\title{
Selecting Features with Group-Sparse Nonnegative Supervised Canonical Correlation Analysis: Multimodal Prostate Cancer Prognosis
}

\author{
Haibo Wang ${ }^{\star}$, Asha Singanamalli, Shoshana Ginsburg, and Anant Madabhushi \\ Department of Biomedical Engineering, Case Western Reserve University, OH 44106 USA \\ $\{$ hxw285, axs1018, sbg52, anantm\}@case.edu
}

\begin{abstract}
This paper presents Group-sparse Nonnegative supervised Canonical Correlation Analysis (GNCCA), a novel methodology for identifying discriminative features from multiple feature views. Existing correlation-based methods do not guarantee positive correlations of the selected features and often need a pre-feature selection step to reduce redundant features on each feature view. The new GNCCA approach attempts to overcome these issues by incorporating (1) a nonnegativity constraint that guarantees positive correlations in the reduced representation and (2) a group-sparsity constraint that allows for simultaneous between- and within- view feature selection. In particular, GNCCA is designed to emphasize correlations between feature views and class labels such that the selected features guarantee better class separability. In this work, GNCCA was evaluated on three prostate cancer $(\mathrm{CaP})$ prognosis tasks: (i) identifying $40 \mathrm{CaP}$ patients with and without 5-year biochemical recurrence following radical prostatectomy by fusing quantitative features extracted from digitized pathology and proteomics, (ii) predicting in vivo prostate cancer grade for $16 \mathrm{CaP}$ patients by fusing T2w and DCE MRI, and (iii) localizing CaP/benign regions on MR spectroscopy and MRI for 36 patients. For the three tasks, GNCCA identifies a feature subset comprising $2 \%, 1 \%$ and $22 \%$, respectively, of the original extracted features. These selected features achieve improved or comparable results compared to using all features with the same Support Vector Machine (SVM) classifier. In addition, GNCCA consistently outperforms 5 state-of-the-art feature selection methods across all three datasets.
\end{abstract}

\section{Introduction}

Availability of multiple data streams presents an opportunity to fuse and combine multimodal biomarkers, for potentially improving performance of predictors of disease diagnosis and prognosis. Canonical correlation analysis (CCA) [1] addresses this multimodal fusion task by attempting to maximize the correlations of the multiple data sources. Supervised multi-view CCA (SMVCCA) [2] combines the principle of CCA

\footnotetext{
* Research was supported by R01CA136535-01, R01CA140772-01, R21CA167811-01, R01DK098503-02, PC120857, and the Ohio Third Frontier Technology development Grant. The content is solely the responsibility of the authors and does not necessarily represent the official views of the NIH.
} 
and linear discriminant analysis (LDA), to find a subspace that maximizes the correlations of multi-view signals and ensures discriminability of provided class labels. Despite its advantages, SMVCCA has some key limitations. First, the CCA components can be negatively correlated, which is less interpretable in practice and hurts the positive dependency between data and their class labels. Secondly, a pre-feature selection step is required in order to reduce redundant features [2]. Thirdly, SMVCCA emphasizes the correlations of all modalities, but neglects modality-specific information.

In this paper, we present Group-sparse Nonnegative supervised CCA (GNCCA), which incorporates (1) nonnegativity and (2) group sparsity constraints to overcome the aforementioned issues of SMVCCA. The nonnegativity is applied onto both the projection and coefficient factor matrices, thus ensuring latent components are positively correlated. Group sparsity allows to simultaneously perform view fusion and within-view feature selection, thus capturing both view-shared and view-specific information. In particular, in order to encourage the association between feature views and class labels, GNCCA keeps the projection section of the label view non-sparse. Although sparse nonnegative CCA has been previously proposed [3], the framework can only calculate the projection of a single view at a time, which has no group sparsity and is difficult to be extended for multiple views. In contrast, GNCCA is based on a more general matrix factorization scheme, which can update all view projections simultaneously.

By ranking the original features based on their values in the projected subspaces [4], GNCCA can be easily used as a multi-modal feature selector, providing an efficient way to interpret the importance of each of the original features for feature-based classification tasks. Unlike the existing feature selection tools, which either neglect view information [4-7] or address only class separability with group lasso [8], GNCCA considers both view association and the discriminability of the selected features.

In this work, we compared GNCCA with 5 related feature selection schemes on three prostate cancer $(\mathrm{CaP})$ prognosis tasks: (i) identifying $40 \mathrm{CaP}$ patients with and without 5-year biochemical recurrence following radical prostatectomy by fusing quantitative features extracted from digitized pathology and proteomics, (ii) predicting in vivo prostate cancer grade for $16 \mathrm{CaP}$ patients by fusing T2 $\mathrm{w}$ and DCE MRI, and (iii) localizing $\mathrm{CaP} /$ benign regions on MR spectroscopy and MRI for 36 patients.

\section{Related Work}

In this section we first describe supervised multi-view CCA (SMVCCA) and then discuss previous work in sparse nonnegative CCA.

Supervised Multi-View Canonical Correlation Analysis (SMVCCA). Provided $n$ data samples $\mathbf{X}$ from $K$ feature views, multi-view CCA [1] seeks a set of linear transformations $\left\{\mathbf{W}_{1}, \mathbf{W}_{2}, \ldots, \mathbf{W}_{K}\right\}$ such that the sum of the correlations of every two views are maximized. In order to ensure class separability, supervised multi-view CCA (SMVCCA) [2] was proposed to simultaneously maximize the correlations of feature views and the correlations between $\mathbf{X}$ and its class labels $\mathbf{Y}$, which yields:

$$
\begin{array}{ll} 
& \arg \max _{\mathbf{W}_{x}, \mathbf{W}_{y}} \operatorname{trace}\left(\mathbf{W}_{x}^{T} \mathbf{C} \mathbf{W}_{x}\right)+2 \operatorname{trace}\left(\mathbf{W}_{x}^{T} \mathbf{X} \mathbf{Y}^{T} \mathbf{W}_{(y)}\right) \\
\text { s.t. } & \mathbf{W}^{T} \mathbf{C}_{d_{x y}} \mathbf{W}=\mathbf{I}, \mathbf{W}_{(1)}^{T} \mathbf{C}_{d_{x y}}^{(11)} \mathbf{W}_{(1)}=\ldots=\mathbf{W}_{(K)}^{T} \mathbf{C}_{d_{x y}}^{(K K)} \mathbf{W}_{(K)}=\mathbf{W}_{(y)}^{T} \mathbf{Y} \mathbf{Y}^{T} \mathbf{W}_{(y)},
\end{array}
$$


where $\mathbf{W}=\left[\mathbf{W}_{(1)}^{T}, \cdots, \mathbf{W}_{(K)}^{T}, \mathbf{W}_{(y)}^{T}\right]^{T}, \mathbf{I}$ is an identity matrix, $\mathbf{C}$ is the covariance matrix of the $K$ views of $\mathbf{X}, \mathbf{C}_{d_{x y}}$ is the block-diagonal section of $\mathbf{C}$, and $\mathbf{C}_{d_{x y}}^{(j j)}$ is the $j^{\text {th }}$ diagonal block of $\mathbf{C}_{d_{x y}}$ [2].

Sparse Nonnegative CCA. Sparse nonnegative CCA, which was first proposed in [3], takes the form:

$$
\begin{aligned}
\max _{\mathbf{w}_{1}, \mathbf{w}_{2}} & \mathbf{w}_{1}^{T} \mathbf{C}_{12} \mathbf{w}_{2}, \\
\text { s.t. } & \left\|\mathbf{w}_{1}\right\|^{2} \leq 1,\left\|\mathbf{w}_{2}\right\|^{2} \leq 1, P\left(\mathbf{w}_{1}\right) \leq c_{1}, P\left(\mathbf{w}_{2}\right) \leq c_{2}, \mathbf{w}_{1} \succeq \mathbf{0}, \mathbf{w}_{2} \succeq \mathbf{0},
\end{aligned}
$$

where $\mathbf{w}_{1}$ and $\mathbf{w}_{2}$ are the two pursued canonical projections; the constraints $\mathbf{w}_{1} \succeq$ $\mathbf{0}, \quad \mathbf{w}_{2} \succeq \mathbf{0}$ enforces that each element in $\mathbf{w}_{1}$ and $\mathbf{w}_{2}$ must be nonnegative. The constraints $\left\|\mathbf{w}_{1}\right\|^{2} \leq 1,\left\|\mathbf{w}_{2}\right\|^{2} \leq 1$ are the convex relaxations of the equality constraints $\left\|\mathbf{w}_{1}\right\|^{2}=1,\left\|\mathbf{w}_{2}\right\|^{2}=1$, which normalize the projections. $P$ is a convex sparsity-inducing penalty with $c_{1}$ and $c_{2}$ being two sparsity controllers. Optimization with respect to $\mathbf{w}_{1}$ and $\mathbf{w}_{2}$ involves iteratively fixing one and solves the other.

\section{Group-sparse Nonnegative supervised CCA (GNCCA)}

GNCCA combines the benefits of SMVCCA and sparse nonnegative CCA by introducing a new group-sparse penalty, relaxing the normalization constraint, and, in particular, encouraging the relevance between features and class labels.

Following the notations in Eq. (1), let $\mathbf{Z}=\left[\mathbf{X}^{T} \mathbf{Y}^{T}\right]^{T}$ denote the stacking of the $n$ $K$-View data samples $\mathbf{X}$ and their labels $\mathbf{Y}$. Correspondingly, let $\mathbf{C}_{x y}$ denote the stacking of the covariance matrix $\mathbf{C}$ and $\mathbf{Y} \mathbf{Y}^{T}$. In order to make GNCCA solvable after integrating group sparsity, the objective of SMVCCA, $\operatorname{trace}\left(\mathbf{W}^{T} \mathbf{C}_{x y} \mathbf{W}\right)$, is equivalently transformed to its Frobenius-Norm: $\|\mathbf{Z}-\mathbf{W H}\|_{F}^{2}$, where $\mathbf{H}$ is the coefficient matrix, and $\mathbf{W}$ is the basis matrix. Based on this reformulation, GNCCA takes the form:

$$
\begin{aligned}
\min & \frac{1}{2}\|\mathbf{Z}-\mathbf{W H}\|_{F}^{2}+\alpha\|\mathbf{H}\|_{F}^{2}+\beta \sum_{k=1}^{K}\left\|\mathbf{W}_{(k)}\right\|_{1, \infty}, \\
\text { s.t. } & \forall\left\|\mathbf{w}_{i}^{(k)}\right\|^{2} \leq 1-\beta, k=1, \cdots, K ; i=1, \cdots, r ; \\
& \forall\left\|\mathbf{w}_{i}^{(y)}\right\|^{2}=1-\beta, \quad i=1, \cdots, r ; \\
& \mathbf{H} \geq \mathbf{0}, \mathbf{W} \geq \mathbf{0},
\end{aligned}
$$

where $\mathbf{W} \in \mathcal{R}^{m \times r}, \mathbf{H} \in \mathcal{R}^{r \times n}$, parameters $\alpha, \beta$ control the relative influence of each penalty term, $K$ is the number of feature views, $r$ is the dimension of reduced representation, $r \ll m$, and \|\|$_{1, \infty}$ refers to $\ell_{1, \infty}$-norm. The nonnegative constraints $\mathbf{H} \geq \mathbf{0}, \mathbf{W} \geq \mathbf{0}$ ensure that both the canonical correlations of training and testing data are positive. The penalty $\|\mathbf{H}\|_{F}^{2}$ is to avoid an arbitrarily large $\mathbf{H}$.

The uniqueness of GNCCA lies in two aspects. First is the group-sparsity penalty on the $K$-View basis $\mathbf{W}_{(1: K)}: \sum_{k=1}^{K}\left\|\mathbf{W}_{(k)}\right\|_{1, \infty}$. Each $\ell_{1, \infty}$-norm is defined by: $\left\|\mathbf{W}_{(k)}\right\|_{1, \infty}$ $=\sum_{i=1}^{r}\left\|\mathbf{w}_{i}^{(k)}\right\|_{\infty}=\left\|\mathbf{w}_{1}^{(k)}\right\|_{\infty}+\cdots\left\|\mathbf{w}_{r}^{(k)}\right\|_{\infty}$, which is the sum of vector $\ell_{\infty}$-norms of its columns. Such $\ell_{1, \infty}$-norm is used to promote as many zero columns as possible in $\mathbf{W}_{(1)}, \cdots \mathbf{W}_{(K)}$, which indicates that only the correlations of the non-zero feature views are maximized. In this way, GNCCA captures both the sharing among modalities 
and the uniqueness of each modality. $\left\|\mathbf{w}_{i}^{(k)}\right\|^{2} \leq 1-\beta$ is to ensure that the correlations are normalized. In particular, we change the relaxation in Eq. (2), $\forall\left\|\mathbf{w}_{i}^{(k)}\right\|^{2} \leq 1$, to be $\forall\left\|\mathbf{w}_{i}^{(k)}\right\|^{2} \leq 1-\beta$. This is because the $\ell_{1, \infty}$ penalty is related to the constraints $\forall\left\|\mathbf{w}_{i}^{(k)}\right\|^{2} \leq 1$. The insight is that for sparse features, the $\ell_{2}$-norm of these features should be smaller than the norms of dense features.

Secondly, we keep the normalization for the label-view basis: $\forall\left\|\mathbf{w}_{i}^{(y)}\right\|^{2}=1-\beta$, which ensures that the selected features are closely correlated with the class labels.

Problem (3) is optimized by adapting the efficient vector-block coordinate descent (BCD) method [9], which updates one column of a factor matrix at each step while fixing all other values. The optimization randomly initializes $\mathbf{W}$ and $\mathbf{H}$, and then updates each vector of $\mathbf{H}, \mathbf{W}_{(1: K)}$ and $\mathbf{W}_{(y)}$ in three steps, until either the objective function is below a preset threshold or the maximum number of iterations has been reached.

Step 1: Fixing $\mathbf{W}$, update each row vector $\mathbf{h}_{i}$. $\in \mathbb{R}^{1 \times n}, i=1, \cdots, r$ as

$$
\mathbf{h}_{i} \leftarrow \arg \min _{\mathbf{h} \geq 0} \frac{1}{2}\left\|\mathbf{R}_{i}-\mathbf{w}_{i} \mathbf{h}\right\|_{F}^{2}+\alpha\|\mathbf{h}\|_{2}^{2},
$$

where $\mathbf{R}_{i}=\mathbf{Z}-\sum_{j=1, j \neq i}^{r} \mathbf{w}_{j} \mathbf{h}_{j}$. This subproblem is solved in a closed form: $\mathbf{h}_{i}$. $\left[\frac{\mathbf{w}_{i}^{T} \mathbf{R}_{i}}{2 \alpha+\left\|\mathbf{w}_{i}\right\|^{2}}\right]_{+}$, where []$_{+}$denote the element-wise projection to nonnegative numbers.

Step 2: Fixing $\mathbf{H}$ and $\mathbf{W}_{(y)}$, update each column vector $\mathbf{w}_{i}^{(k)}, k=1, \cdots, K$ as

$$
\mathbf{w}_{i}^{(k)} \leftarrow \arg \min _{\mathbf{w} \geq 0,\|\mathbf{w}\|^{2} \leq 1-\beta} \frac{1}{2}\left\|\mathbf{R}_{i}^{(k)}-\mathbf{w}^{(k)} \mathbf{h}_{i} \cdot\right\|_{F}^{2}+\beta\left\|\mathbf{w}^{(k)}\right\|_{\infty},
$$

where $\mathbf{R}_{i}^{(k)}=\mathbf{Z}^{(k)}-\sum_{j=1, j \neq i}^{r} \mathbf{w}_{j}^{(k)} \mathbf{h}_{j}$. This subproblem can be efficiently solved by first finding the solution $\mathbf{w}_{i}^{(k) *}$ of the problem described in [10], then normalizing $\mathbf{w}_{i}^{(k) *}$ such that $\left\|\mathbf{w}_{i}^{(k) *}\right\|^{2} \leq 1-\beta$, and finally updating $\mathbf{w}_{i}^{(k) *}$ as $\mathbf{w}_{i}^{(k) * *}=\left[\frac{\mathbf{R}_{i}^{(k)} \mathbf{h}_{i \cdot}^{T}}{\left\|\mathbf{h}_{i \cdot}\right\|^{2}}\right]_{+}-\mathbf{w}_{i}^{(k) *}$.

Step 3: Fixing $\mathbf{W}_{(1: K)}$ and $\mathbf{H}$, update $\mathbf{W}_{(y)}$ as

$$
\mathbf{w}_{i}^{(y)} \leftarrow \arg \min _{\mathbf{w} \geq 0,\|\mathbf{w}\|^{2}=1-\beta} \frac{1}{2}\left\|\mathbf{R}_{i}^{(y)}-\mathbf{w}^{(y)} \mathbf{h}_{i}\right\|_{F}^{2},
$$

where $\mathbf{R}_{i}^{(y)}=\mathbf{Z}^{(y)}-\sum_{j=1, j \neq i}^{r} \mathbf{w}_{j}^{(y)} \mathbf{h}_{j}$. The solution of this subproblem is to first update $\mathbf{w}$ as $\mathbf{w}_{i}^{(y)} \leftarrow\left[\frac{\mathbf{R}_{i}^{(y)} \mathbf{h}_{i .}^{T}}{\left\|\mathbf{h}_{i \cdot}\right\|^{2}}\right]_{+}$, and then normalize $\mathbf{w}_{i}^{(y)}$ by $\mathbf{w}_{i}^{(y)} \leftarrow \frac{\mathbf{w}_{i}^{(y)}}{\left\|\mathbf{w}_{i}^{(y)}\right\|} \sqrt{1-\beta}$.

The above optimization result of GNCCA leads to:

$$
\mathbf{Z}=\left[\begin{array}{c}
\mathbf{X} \\
\mathbf{Y}
\end{array}\right] \approx\left[\begin{array}{c}
\mathbf{W} \\
\mathbf{W}_{y}
\end{array}\right] \mathbf{H}
$$

which provides two approximate relationships: $\mathbf{X} \approx \mathbf{W H}$ and $\mathbf{Y}^{T} \approx \mathbf{H}^{T} \mathbf{W}_{y}^{T}$. The former introduces a lower feature representation $\mathbf{H}$ in the reduced feature space $\mathbf{W}$ while the latter models how the lower representation $\mathbf{H}$ is regressed to class labels $\mathbf{Y}^{T}$. Given these two relationships, we rank the original $j$ th feature according to the Variable Importance in the Projections (VIP) score [4], which determines the importance of original features based on their values in the projected subspace:

$$
\pi_{j}=\sqrt{\frac{m \sum_{i=1}^{r}\left\|\mathbf{w}_{y}\right\|_{2}^{i} \mathbf{h}_{i} \cdot \mathbf{h}_{i \cdot}^{T}\left(\frac{w_{j i}}{\left\|\mathbf{w}_{i}\right\|}\right)^{2}}{\sum_{i=1}^{r}\left\|\mathbf{w}_{y}\right\|_{2}^{i} \mathbf{h}_{i} \cdot \mathbf{h}_{i}^{T} .}},
$$


where $w_{y}^{i} \in \mathbf{W}_{y}$ is column entry of $\mathbf{W}_{y}$. To automatically determine the number of selected features, we define a threshold $\sigma: 0<\sigma<1$. Only top features whose scores are no smaller than $\sigma \times \pi_{\max }$ will be selected, where $\pi_{\max }$ is the largest VIP score. For simplicity, we call the GNCCA based feature selector GNCCA-VIP.

\section{Experiments}

\subsection{Data Sets}

To evaluate GNCCA-VIP, we chose three unique datasets that enabled us to address three of the most relevant problems in the disease domain of prostate cancer $(\mathrm{CaP})$, i.e., predict biochemical recurrence, identifying $\mathrm{CaP}$ grade and localizing $\mathrm{CaP}$. GNCCAVIP is applied to select the most important ones from extracted imaging/non-imaging features such that the selected features can be used to better address the associated task of $\mathrm{CaP}$ prognosis.

We evaluate the proposed method by using D1 [2], D2 [2] and D3 [11] datasets (see Table 1. Representative images from D1 and D2 are shown in Fig. 1 D1 contains 21 $\mathrm{CaP}$ patients who experienced and $19 \mathrm{CaP}$ patients who did not experience biochemical recurrence within 5 years of radical prostatectomy. For each patient, 242 histological features and 650 proteomic profiling values are extracted. D2 contains 33 MRI slices from $16 \mathrm{CaP}$ patients. 22 slices have prostate cancer with a Gleason grade [12] of 3, while the other 11 have a grade of 4 . On each slice, 112 textural features and $38 \mathrm{ki}$ netic features are extracted from T2w and DCE MRI, respectively. D3 contains 2901 $\mathrm{CaP} /$ non-CaP voxels from 36 patients that were annotated by an expert clinician. From within each voxel, 6 MRS features and 58 MRI features are extracted.

Table 1. Summary of the benchmark data sets

\begin{tabular}{|c|c|c|c|c|}
\hline Data set & \#Views & \#Features & $\#$ Patients & Task \\
\hline D1 [2] & 2 & $892(242+650)$ & 40 & predict biochemical recurrence \\
\hline D2 [2] & 2 & $150(112+38)$ & 16 & identify CaP grade \\
\hline D3 [11] & 2 & $64(6+58)$ & 36 & localize CaP \\
\hline
\end{tabular}

\subsection{Experimental Settings}

A Leave- $M$-Patients-Out cross validation scheme was used in conjunction with a linear Support Vector Machine (SVM) classifier [13] to evaluate the feature selection performance of GNCCA-VIP with the following comparative strategies: i) All-features, ii) FisherScore [5], iii) T-test [6], iv) mRMR [7], v) PCA-VIP [4], and vi) SMVCCAVIP [2]. The parameter $c$ of SVM is set to be $c=1$. The critical parameters of GNCCA, including $\beta, r$ and $\sigma$, are determined via Leave-1-Patient-out cross validation. The controller of $\|\mathbf{H}\|_{F}^{2}, \alpha$, is fixed at 0.1 . The number of patients left out, $M$, varies according to the number of total patients in each dataset.

Each experiment was repeated 50 times, and the average classification accuracy as well as variations were reported. All the experiments were conducted within a Matlab environment on a 64-bit Linux machine with 4-core CPU and 4G memory. 


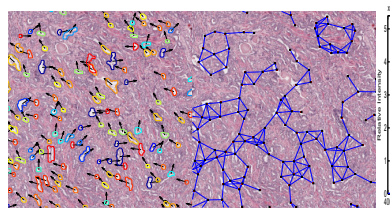

(a)

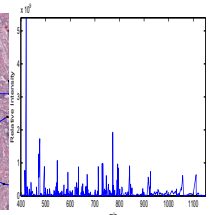

(c)

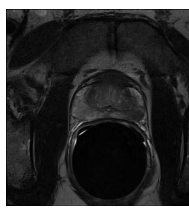

(d)

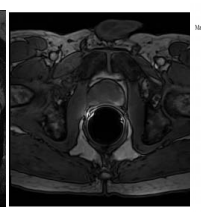

(e)

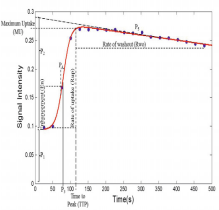

(f)

Fig. 1. D1 histology dataset: (a) textural features extracted on excised gland orientations, (b) structural features, and (c) Proteomic profiling values. D2 dataset: (d) T2w MRI, (e) DCE MRI, and (f) Kinetic curve extracted from DCE MRI.

\subsection{Experiment 1: GNCCA-VIP versus Other Feature Selection Schemes}

Fig. 2] illustrates the classification results obtained with the compared feature selection approaches in conjunction with linear SVM. Fig. 3 shows the corresponding number of selected features with each selection approach. On D1, GNCCA-VIP achieves better classification than All-features while selecting only $2 \%$ of the features (18 out of 892). Although other methods (except mRMR) select fewer features, their classification results are obviously worse. On D2, GNCCA-VIP obtains the highest classification results with only selecting an average of 2 features from 150 features. On D3, GNCCA-VIP selects an average of 13 features (out of the 64 features) and achieves the same classification result as using all features.

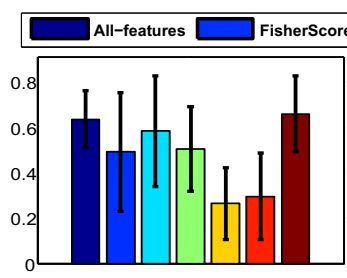

(a) D1

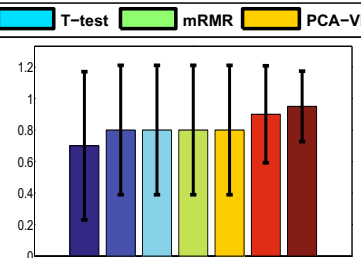

(b) D2

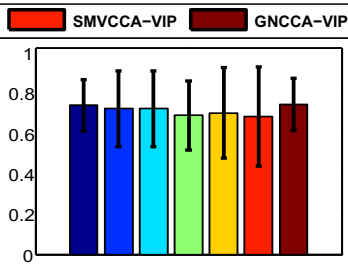

(c) D3

Fig. 2. Comparing the prognosis accuracies (and standard deviations (STD)) with the features selected via All-features, FisherScore, T-test, mRMR, PCA-VIP, SMVCCA-VIP and GNCCAVIP on the three dataset. The same linear SVM is used as the classifier here. The proposed GNCCA-VIP approach achieves highest accuracies (with lowest STDs) in all the prognosis tasks.

The results also reveal that GNCCA-VIP is likely to select a comparable number of features from each modality. For example, on D3, GNCCA-VIP is most likely to select all $6 \mathrm{MRS}$ features and then select 6 or $7 \mathrm{MRI}$ features that are related to the $6 \mathrm{MRS}$ features. By contrast, the other feature selection methods are likely to only select MRI features, which is clearly a bias towards the dominant MRI features. All these results demonstrate the ability of GNCCA-VIP in identifying features that are discriminative of provided class labels and leads to better view association. 


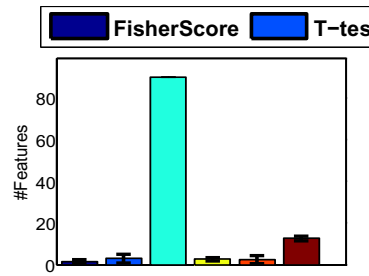

(a) D1

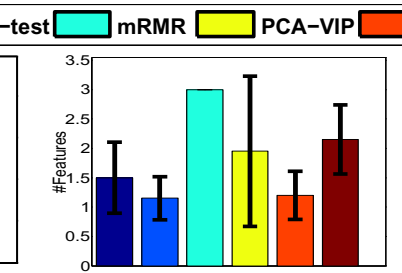

(b) D2

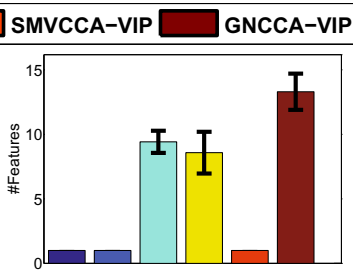

(c) D3

Fig. 3. The average number of features (STD) adaptively selected via FisherScore, T-test, mRMR, PCA-VIP, SMVCCA-VIP and GNCCA-VIP on achieving the reported prognosis accuracies in Fig. 2. Note that at each time mRMR selects the same number of features on D1 and D2.

\subsection{Experiment 2: Parameter Sensitivity of GNCCA-VIP}

Fig. 4 shows the sensitivity of GNCCA-VIP to four critical parameters that must be manually tuned: i) the group-sparsity controller $\beta$ in Eq. (3), ii) the intrinsic dimensionality $r$ in Eq. (3), iii) the threshold $\sigma$ that controls the number of selected features, and iv) $M$, the number of patients left out. The D1 dataset is used for this experiment. As we can see, GNCCA-VIP is relatively insensitive to $\beta, r$ and $\sigma$. As $M$ increases, indicating that more patients are being used as testing samples and fewer subjects are being retained for training purposes, all the classification results gradually degrade. However, GNCCA-VIP consistently leads to favourable classification result compared to using all features.

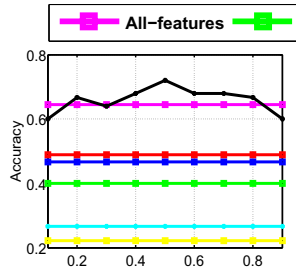

(a) varying $\beta$

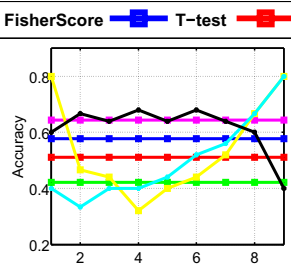

(b) varying $r$

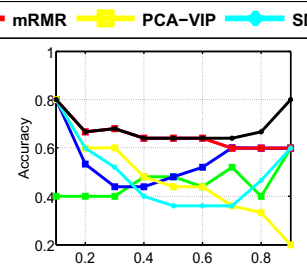

(c) varying $\sigma$

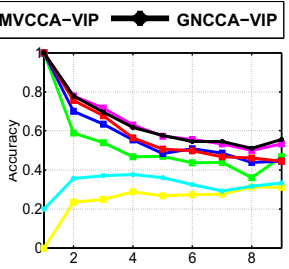

(d) varying $M$

Fig. 4. Testing the influence of parameters $\beta, r, \sigma$ and $M$. (a) varying $\beta$ from 0.1 to 0.9 by fixing $r=1, \sigma=0.9, M=1$. (b) varying $r$ from 1 to 9 by fixing $\beta=0.7, \sigma=0.9, M=5$. (c) varying $\sigma$ from 0.1 to 0.9 by fixing $\beta=0.7, r=2, M=1$. (d) varying $M$ from 1 to 9 by fixing $r=1, \sigma=0.7, \beta=0.9$. Note that the $\mathrm{D} 1$ dataset is used for this experiment.

\section{Concluding Remarks}

This paper presents GNCCA-VIP, a novel method for multimodal feature selection that incorporates nonnegativity and group-sparse constraints into supervised CCA. The nonnegativity ensures that latent components are always positively correlated. Group sparsity incorporates strengths from both between- and within- view sparsity, thus allowing 
for simultaneous view association and single-view feature selection. In our experiments, the features selected via GNCCA-VIP result in improved classification performance in $\mathrm{CaP}$ prognosis prediction compared to using either the entire feature set or using other state-of-the-art feature selection schemes. Hence, this approach may be a better choice in the context of combining multi-scale, multi-modal data.

\section{References}

1. Kettenring, J.R.: Canonical analysis of several sets of variables. Biometrika 58(3), 433-451 (1971)

2. Singanamalli, A., Wang, H., et al.: Supervised multi-view canonical correlation analysis: Fused multimodal prediction of disease diagnosis and prognosis. In: SPIE Medical Imaging, vol. 9038 (2014)

3. Witten, D.M., Tibshirani, R.J.: Extensions of sparse canonical correlation analysis with applications to genomic data. Statistical Applications in Genetics and Molecular Biology 8(1), 1-27 (2009)

4. Ginsburg, S., Tiwari, P., Kurhanewicz, J., Madabhushi, A.: Variable ranking with pca: Finding multiparametric mr imaging markers for prostate cancer diagnosis and grading. In: Madabhushi, A., Dowling, J., Huisman, H., Barratt, D. (eds.) Prostate Cancer Imaging 2011. LNCS, vol. 6963, pp. 146-157. Springer, Heidelberg (2011)

5. Duda, R.O., Hart, P., Stork, D.: Pattern classification. Journal of Classification 24(2), 305307 (2007)

6. Student: The problem error of a mean. Biometrika 6, 1-25 (1908)

7. Peng, H., Long, F., Ding, C.: Feature selection based on mutual information: Criteria of maxdependency, max-relevance, and min-redundancy. IEEE Transactions on Pattern Analysis and Machine Intelligence (T-PAMI) 27(8), 1226-1238 (2005)

8. Ye, J., Liu, J.: Sparse methods for biomedical data. SIGKDD 14(1), 4-15 (2012)

9. Jingu Kim, R.M., Park, H.: Group sparsity in nonnegative matrix factorization. In: SIAM International Conference on Data Mining (SDM), pp. 851-862 (2012)

10. Duchi, J., et al.: Efficient projections onto the 11-ball for learning in high dimensions. In: The 25th International Conference on Machine Learning (ICML), pp. 272-279 (2008)

11. Tiwari, P., et al.: Multimodal wavelet embedding representation for data combination (maweric): integrating magnetic resonance imaging and spectroscopy for prostate cancer detection. NMR in Biomedicine 25(4), 607-619 (2012)

12. Gleason, D.F.: The veteran's administration cooperative urologic research group: histologic grading and clinical staging of prostatic carcinoma. In: Urologic Pathology: The Prostate, pp. 171-198 (1977)

13. Cortes, C., Vapnik, V.: Support-vector networks. Machine Learning 20(3), 273-297 (1995) 